\title{
Late-Onset Hypertrophic Pyloric Stenosis in a 14-Weeks-Old Full Term Male Infant
}

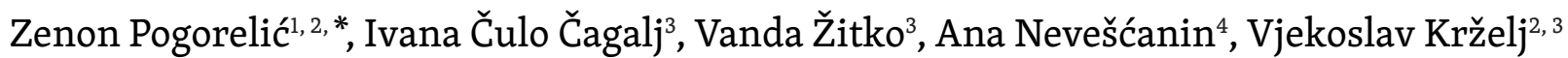

\begin{abstract}
Background: Hypertrophic pyloric stenosis is the most common cause of gastric outlet obstruction in infants, and classically presents at 2 to 6 weeks of age. Delayed presentation is an extremely rare occurrence after early infancy.

Case report: A 14-weeks-old full term male infant presented with non-bilious vomiting, dehydration and hypocloremic metabolic alkalosis. Abdominal ultrasonography revealed tubular mass $20 \mathrm{~mm}$ in lenght. Because of unusual age, diagnosis was confirmed with upper gastrointestinal contrast study. Laparoscopic pyloromyotomy was performed. After surgery the child was free of symptoms, had gained weight, and was tolerating a regular diet.

Message: Despite the age hypertrophic pyloric stenosis should be kept in mind in any child who presents with non-bilious vomiting.
\end{abstract}

\section{KEYWORDS}

hypertrophic pyloric stenosis; children; infants; late presentation; non-bilious vomiting

\section{AUTHOR AFFILIATIONS}

${ }^{1}$ Department of Pediatric Surgery, University Hospital of Split, Split, Croatia

2 University of Split, School of Medicine, Split, Croatia

3 Department of Pediatrics, University Hospital of Split, Split, Croatia

${ }^{4}$ Department of Anesthesiology, Reanimatology and Intensive Care, University Hospital of Split, Split, Croatia

* Corresponding author: Department of Pediatric Surgery, Split University Hospital, Spinčićeva 1, 21000 Split, Croatia; e-mail: zpogorelic@gmail.com

Received: 24 February 2019

Accepted: 27 March 2019

Published online: 26 July 2019

Acta Medica (Hradec Králové) 2019; 62(2): 82-84

https://doi.org/10.14712/18059694.2019.108

(c) 2019 The Authors. This is an open-access article distributed under the terms of the Creative Commons Attribution License (http://creativecommons.org/licenses/by/4.0), which permits unrestricted use, distribution, and reproduction in any medium, provided the original author and source are credited. 


\section{INTRODUCTION}

Hypertrophic pyloric stenosis is a common infantile disorder with an incidence of 1.5 to 4 per 1000 live births. The disease classically presents at 2 to 6 weeks of age often in a previously healthy infant, with peak onset at week 4 (1). It is more common in males than females and in infants born preterm as compared with those born at term $(1,2)$.

Despite more than a century pass after first successful treatment of this condition, its etiology still remains incompletely understood. Infants typically present with projectile vomiting associated with symptoms of failure to thrive and metabolic alkalosis. An olive-like mass palpable in the right upper abdominal quadrant is being reported less frequently because of earlier diagnosis by ultrasound. Nowadays hypertrophic pyloric stenosis is generally corrected through laparoscopic pyloromyotomy (3).

\section{CASE REPORT}

A 14-weeks-old full term male infant presented to our hospital with history of non-bilious vomiting. The child occasionally had vomited non-bilious content for about 20 days. There was no history of abdominal pain, visible abdominal tumor, abdominal distension or melena. One week prior to hospitalization he started to vomit more frequently, and last days he vomited after every meal and it became progressive and projectile. Before admission there was admixture of blood in vomit. On physical examination mild dehidration was found. His vital signs were within normal limits. He had no abdominal distension. Abdomen was soft and non-tender. There was a small vague swelling palpable in the right hypochondrium. Hypochloremic metabolic alkalosis was found. Other biochemical and hematological tests were within normal limits. On

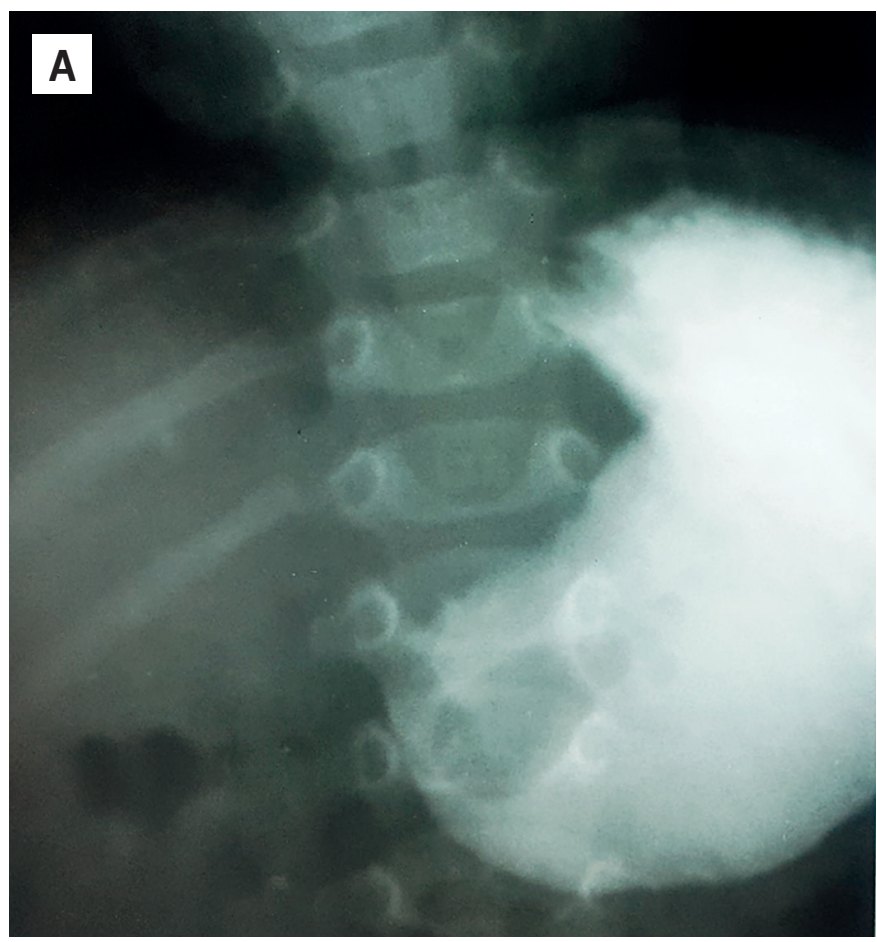

ultrasonography a tubular mass measuring $7.5 \mathrm{~mm}$ in thickness and $26 \mathrm{~mm}$ in length was found. Although child has typical clinical presentation and although he met sonographic criteria for hypertrophic pyloric stenosis, because of unusual age, upper gastrointestinal contrast study was performed. Contrast study reveals a distended stomach with shouldering and string sign with no passage of the contrast from stomach to the duodenum (Fig. 1A). After resuscitation and correction of water and electrolytes disturbances the patient was transferred to pediatric surgeon. Laparoscopy was performed through three 3-mm ports. The pylorus was extremely hypertrophied and firm (Fig. 1B). Laparoscopic pyloromyotomy was done, and the mucosa was seen to pout out nicely. Oral intake was initiated few hours after surgery and the patient was discharged in the third postoperative day without complications. At follow-up, the child was free of symptoms, had gained weight, and was tolerating a regular diet.

\section{DISCUSSION}

Hypertrophic pyloric stenosis is typically a disease of 2- to 6-week-old infants with progressive, non-bilious vomiting; its peak occurrence is at 3-5 weeks of age (1-3).

In the case described here, the patient showed feeding difficulty very late, and feeding intolerance worsened. He presented with non-bilious vomiting at 14th week of life, which is very uncommon age for hypertrophic pyloric stenosis. This delayed presentation with unawareness of the hypertrophic pyloric stenosis lead to delayed diagnosis. In the beginning, although he had typical symptoms, gastroenteritis or gastroesophageal reflux was suspected. After diagnostic procedures diagnosis of pyloric stenosis was established and confirmed at the surgery. Although the exact etiology is still unknown, pyloric muscle thickening does not start before birth. The muscle thickening starts

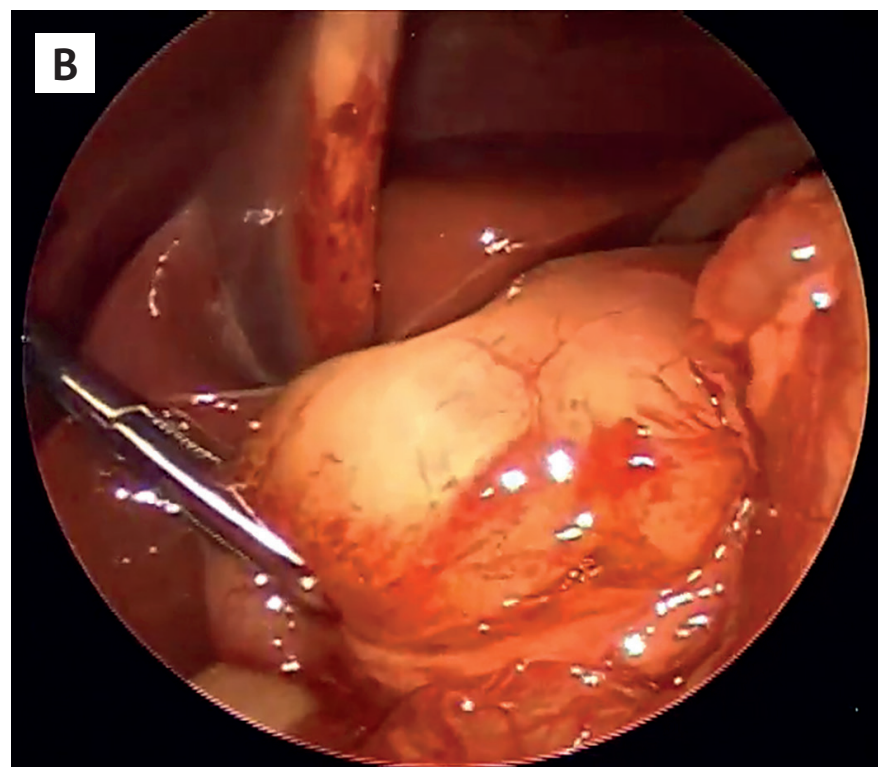

Fig. 1 Hypertrophic pyloric stenosis in a 14-weeks-old male infant. A) Contrast study revealing a distended stomach with no passage of contrast to the duodenum; B) Intraoperative finding on laparoscopy-hypertrophied pylorus. 
after birth and the degree of muscle hypertrophy will determine the age of onset of the vomiting (4). In literature there are just few case reports where delayed presentation after typical age was reported, with age range from 5 months to 5 years (4-8). Late onset presentation gives the condition an acquired origin rather than congenital one. Despite the age hypertrophic pyloric stenosis should be kept in mind in any child who presents with non-bilious vomiting.

\section{REFERENCES}

1. Jobson M, Hall NJ. Contemporary management of pyloric stenosis. Semin Pediatr Surg 2016; 25(4): 219-24.

2. Kapoor R, Kancherla V, Cao Y, et al. Prevalence and descriptive epidemiology of infantile hypertrophic pyloric stenosis in the United
States: A multistate, population-based retrospective study, 19992010. Birth Defects Res 2019; 111(3): 159-69.

3. El-Gohary Y, Abdelhafeez A, Paton E, Gosain A, Murphy AJ. Pyloric stenosis: an enigma more than a century after the first successful treatment. Pediatr Surg Int 2018; 34(1): 21-7.

4. Al-Mayoof AF, Doghan IK. Late onset infantile hypertrophic pyloric stenosis. Journal of Pediatric Surgery Case Reports 2018; 30: $22-4$.

5. Tiao MM, Huang HC, Shieh CS, Huang CB, Liang CD. Infantile hypertrophic pyloric stenosis in a 5-month-old baby: case report. Chang Gung Med J 2000; 23: 442-5.

6. Mahalik S, Prasad A, Sinha A, Kulshrestha R. Delayed presentation of hypertrophic pyloric stenosis: a rare case. J Pediatr Surg 2010: 45: e9-e11

7. Wolf LL, Nijagal A, Flores A, Buchmiller TL. Late-onset hypertrophic pyloric stenosis with gastric outlet obstruction: case report and review of the literature. Pediatr Surg Int 2016; 32: 1013-6.

8. Martínez-Criado Y, Aspiazu D, Cabrera R, De Agustín JC. Delayed presentation of hypertrophic pyloric stenosis in infants: an unusual case. An Pediatr (Barc) 2012; 76(3): 169-70. 